\title{
FEATURE EXTRACTION METHODS FOR COLOR IMAGE SIMILARITY
}

\author{
R.Venkata Ramana Chary ${ }^{1}$, Dr.D.Rajya Lakshmi ${ }^{2}$ and Dr. K.V.N Sunitha ${ }^{3}$ \\ ${ }^{1}$ Padmasri Dr.B.V Raju Institute of Technology, Hyderabad, India \\ rvrcharyegmail.com \\ ${ }^{2}$ GITAM Institute of Technology, Visakhapatnam, India \\ rdavulurieyahoo.com \\ ${ }^{3}$ G. Narayanamma Institute of Technology and Science Hyderabad, India \\ $\mathrm{k} \cdot \mathrm{v} \cdot \mathrm{n}$.sunitha@gmail.com
}

\begin{abstract}
In this paper we concentrated on image retrieval system in early days many user interactive systems performed with basic concepts but such systems are not reaching to the user specifications and not attracted to the user so a lot of research interest in recent years with new specifications, recent approaches have user is interested in friendly interacted methods are expecting, many are concentrated for improvement in all methods. In this proposed system we focus on the retrieval of images within a large image collection based on color projections and different mathematical approaches are introduced and applied for retrieval of images. before Appling proposed methods images are sub grouping using threshold values, in this paper $R G B$ color combinations considered for retrieval of images, in proposed methods are implemented and results are included, through results it is observed that we obtaining efficient results comparatively previous and existing.
\end{abstract}

\section{KEYWORDS}

Color combination, threshold, Retrieval, Query, image Database, mean, standard deviation, median, features, semantic, extraction.

\section{INTRODUCTION}

The term content based image retrieval (CBIR)[1] is the application of computer vision techniques to the image related problems. Experiments into automatic retrieval of images from a large collection database are based on the colors, shapes and texture and image features [2]. At current stage, there is a gap between low-level features of the retrieval system and the high-level semantic[6] concepts of the user, called semantic gap. Compared with text-IR, this problem result in very poor performance of CBIR system .All techniques and algorithms that are used originate from fields such as statistics, pattern recognition[4][5] and computer vision.

Content-Based Image Retrieval (CBIR) is according to the user-supplied in the bottom characteristics, directly find out images containing specific content from the image library The basic process: First of all, do appropriate pre-processing of images like size and image transformation and noise reduction is taking place, and then extract image characteristics needed from the image according to the contents of images to keep in the database. When we retrieve to identify the image, extract the corresponding features[6][7] from a known image and then retrieve the image database to identify the images which are similar with it, also we can give some of the characteristics based on a query requirement, then retrieve out the required images based on the given suitable values. In the whole retrieval process, feature extraction is essential; it is closely related to all aspects of the feature, such as color, shape, texture and space. 


\subsection{Color Image}

A color image is a combination of some basic colors. In MATLAB breaks each individual pixel of a color image (termed 'true color') down into Red, Green and Blue values. We are going to get as a result, for the entire image is 3 matrices, each one representing color features. The three matrices are arranging in sequential order, next to each other creating a 3 dimensional $m$ by $n$ by 3 matrixes. An image which has a height of 5 pixels and width of 10 pixels the resulting in MATLAB would be a 5 by 10 by 3 matrixes for a true color image.

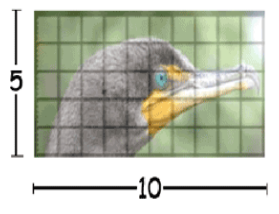

Original Color Image

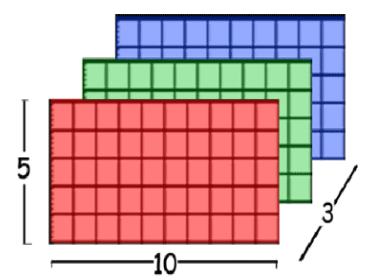

Matlab RGB Matrix

Figure 1. Color image and RGB matrix.

\subsection{Color Panel}

The following picture showing color panels projecting the color components in image is representing in following pictures 2 and 3.

$\mathrm{R}=\mathrm{RGB}(:,:, 1) ; \mathrm{G}=\mathrm{RGB}(:,:, 2) ; \mathrm{B}=\mathrm{RGB}(:,:, 3)$;

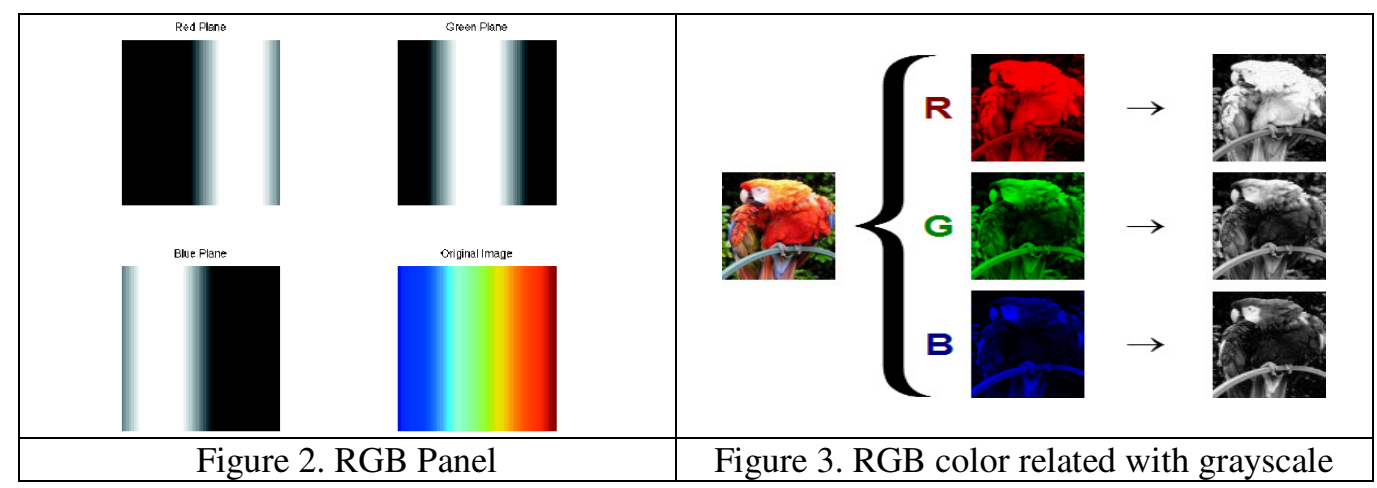

\section{Problem Description}

In image retrieval system for searching, browsing, and retrieving images from a large database of images [14]. Most conventional and common methods of image retrieval utilize some method of adding metadata such as tokens, captioning, keywords, or descriptions to the images so that retrieval can be performed over the annotation words. Some systems are working with lower level features, Manual image annotation is time-consuming, laborious and expensive. To address this, many researchers are proposed on automatic[13] user friendly image retrievals using different methods.

Content-based means that the search will analyze the actual contents of the image. The term 'content' in this context might refer to colors, shapes, textures, or any other information that can be derived from the image itself. Without the ability to examine image content, searches must rely on metadata such as captions or keywords, which may be laborious or expensive to produce. In this paper proposed methods are providing the best solution in large image set. 


\section{Proposed System}

Proposed system uses combinations of color feature to overcome the problem description.

$>$ Proposed system is implemented and concentrated on visual contents of an image such as color, shape, texture and spatial layouts.

$>$ Proposed system selected 10000 image databases with common feature values.

$>$ Proposed system extracted all images features separately $R, G, B$ values for problem solving.

$>$ Proposed system implemented features like color histogram, color projections

$>$ Mathematical approaches like mean, median and standard deviation are proposed for efficient retrieval

Proposed work provides platform to extract images from the database using user query method.

\section{WORK IMPLEMENTATION}

\subsection{Image retrieval is implemented in the following steps}

Image retrieval is implemented in two different phases. One is new image insertion with features in database and other one is searching a new image in available database.

Step 1:- All ten thousand images are taken in to working directory of MATLAB.

Step 2:- Using MATLAB programming all image features in R, G, B color projection values are extracted and stored in database using specified programming methods.

Step 3:-Threshold calculation is taken for categorizing the images into a similar feature groups. In this step, threshold value is computed based on the histogram calculation.

If the image is a color image it will convert into gray color when calculated by the sum of all bins in image histogram. Figure 4 is one example for threshold values of images.

following steps computes the specifying threshold for image

ifisrgb(Image) GImage=rgb2gray(Image);

p12=imhist(GImage); threshold=sum(sum(p12))

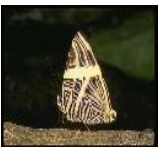

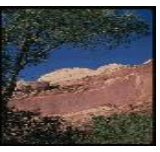

10880
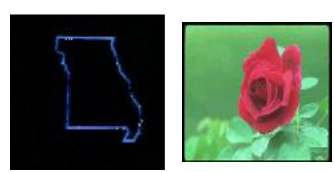

12288

Figure 4 .

Using these methods, all images are categorizing into $\mathrm{N}$ number of Groups

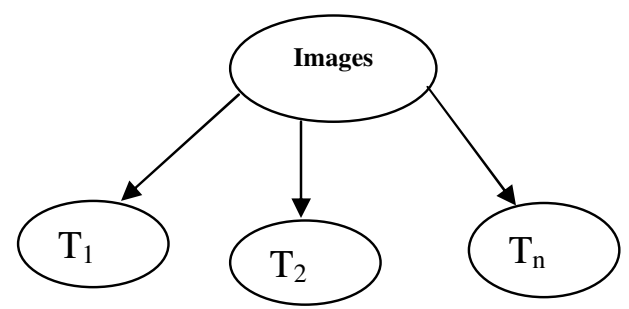


Step 4:-In usual methods, image color values are storing in matrix form. Using Image matrix, all R, G, B components in image are extracted and separated into three different array forms.

( Red, Green, Blue )

$\operatorname{Red}(\mathrm{M}, \mathrm{J}) \quad=\operatorname{Image}(\mathrm{M}, \mathrm{J}, 1)$;

Green $(\mathrm{M}, \mathrm{J}) \quad=\operatorname{Image}(\mathrm{M}, \mathrm{J}, 2)$;

Blue $(\mathrm{M}, \mathrm{J}) \quad=\operatorname{Image}(\mathrm{M}, \mathrm{J}, 3)$;

Step 5 :- Using the feature vectors, each image color wise means are computed . In this method row and overall image mean are computed and stored into the database. Based on all this features different computing methods are formulated.

mean_r=mean2(Red);

mean_g=mean2(Green);

mean_b=mean2(Blue);

median_r=median ( (median (Red))');

median_g=median ( (median (Green)) ') ;

median_b=median ( (median (Blue))' ) ;

std_r $=\operatorname{std}((\operatorname{std}(\operatorname{Red}, 0,1)) ', 0,1)$;

std_g=std ( ( std $($ Green, 0,1$)) ', 0,1)$;

std_b=std $(($ std $($ Blue $, 0,1)) ', 0,1)$;

Step 6:- Query image is selecting based on user choice and verifying threshold value if the threshold values are equal and then proposed methods are implementing

Step 7:- Using the image feature vectors various retrieval methods are proposed. In each method, two different working group sets are identified. One is large image set group and second one is small set of image group.

All Images $=\left\{T_{1}, T_{2}, T_{3} \ldots \ldots T_{n}\right\}$

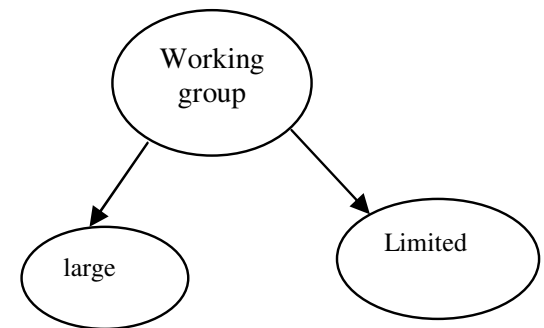

Large $=\mathrm{T}_{\text {equalent set }}\{\mathrm{T}$ is threshold value and all images belongs to same $\mathrm{T}$ group $\}$

Limit $=\mathrm{T}_{\text {equalent set } \text { with less images }}$

\subsection{Proposed Method (PM)}

In each method, two image feature values are verified from available database files, after that comparing equalities of query image (QI) and target image (TI). TI may be similar or equal or not equal. So based on the difference factor(DF), images are identified.

$\mathrm{DF}=$ Constant $\{$ this value is proposed by user $\}$

PM 1:- Selecting only one color mean value from image vector database means are compared in the range of DF.

PM 2:- Selecting two colors mean values from the image feature database if the means are equal or similar values within the range of DF. Two colors mean are like RG, RB, and GB.

PM 3:- Selecting three colors mean values from the image feature database if the means are equal or similar values within the range of DF. 
PM 4:- Selecting three color median values from the image feature database if the medians are equal or similar values within the range of DF

PM 5:- Selecting the different color standard deviation methods (SD) are applied for image comparisons. This method has given a good result comparatively. Other proposed method results are discussed in section 5 .

\section{RESULTS}

\subsection{Experimental Results}

Query is applied by using mean values

\begin{tabular}{|c|c|c|c|c|c|}
\hline p.name & R_mean & Picture & p.name & R_mean & Picture \\
\hline 998.jpg & 63.36859 & & 992.jpg & 83.37225 & \\
\hline 997.jpg & 65.08289 & & 991.jpg & 84.91315 & \\
\hline 995.jpg & 75.65936 & & 996.jpg & 86.83167 & \\
\hline 993.jpg & 82.29895 & & 994.jpg & 110.7877 & \\
\hline
\end{tabular}

In above figure 5 it is showing that if the image is searching based on red_mean type top 8 pictures are displaying in table order .in above example DF value is 25 from pic 993.jpg to $R \_m e a n$ value is 75.65 remaining pictures -25 and +25 mean values considered .

\begin{tabular}{|l|c|c|c|c|c|c|c|}
\hline \multicolumn{10}{|c|}{ Table 1: Mean methods on 8 pictures and retrieval ranking } \\
\hline PIC & $\begin{array}{l}\text { R } \\
\text { MEAN }\end{array}$ & G & B & $\begin{array}{l}\text { RG } \\
\text { AVG }\end{array}$ & RB & GB & RGB \\
\hline 991.jpg & 6 & 6 & 5 & 6 & 4 & 6 & 4 \\
\hline 992.jpg & 5 & 5 & 7 & 5 & 8 & 5 & 8 \\
\hline 993.jpg & 4 & 7 & 8 & 7 & 6 & 7 & 7 \\
\hline 994.jpg & 8 & 8 & 2 & 1 & 5 & 8 & 6 \\
\hline 995.jpg & 3 & 3 & 1 & 3 & 2 & 3 & 2 \\
\hline 996.jpg & 7 & 4 & 6 & 4 & 7 & 4 & 5 \\
\hline 997.jpg & 2 & 1 & 3 & 1 & 1 & 1 & 1 \\
\hline 998.jpg & 1 & 2 & 4 & 2 & 3 & 2 & 3 \\
\hline
\end{tabular}

In the Table 1 is showing that picture names and color means of R,G,B ,two color combination averages and final RGB is calculated values represented 8 images in above table all proposed methods are applied based on that ranks are showing .

In the above tables based on the color mean combination methods results are showing that almost 75 to $90 \%$ of picture is looking similar.At the same time we can notify that the results for single color to combination of colors features if combining similar images are coming together .this we can observe through ranking( 1 to 8$)$, in this observation we can conclude that 
instead of using single color projection we can select different combinations of color methods applying we can achieve good performance.

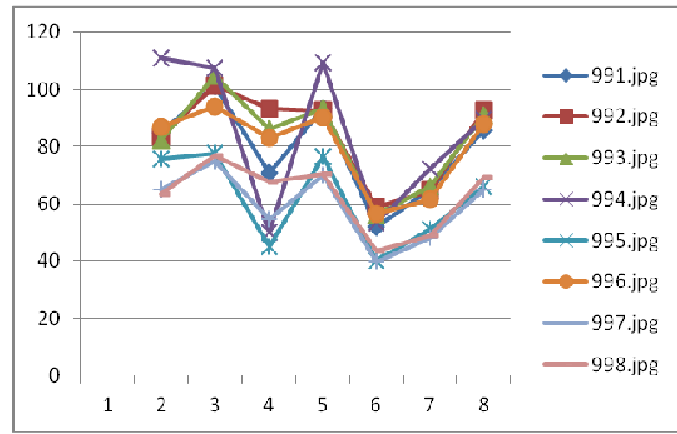

Figure 6.

In above graph(Figure 6) images relations in various proposed methods are indicated . (2 is r_mean,3 is g_mean,4 is b_mean,5 is rg_avg, 6is rb_avg,7is gb_avg,8 is rgb_avg means.)

5.2 Second proposed method based on median .

Median red applied for selected set of images in that following order is performed

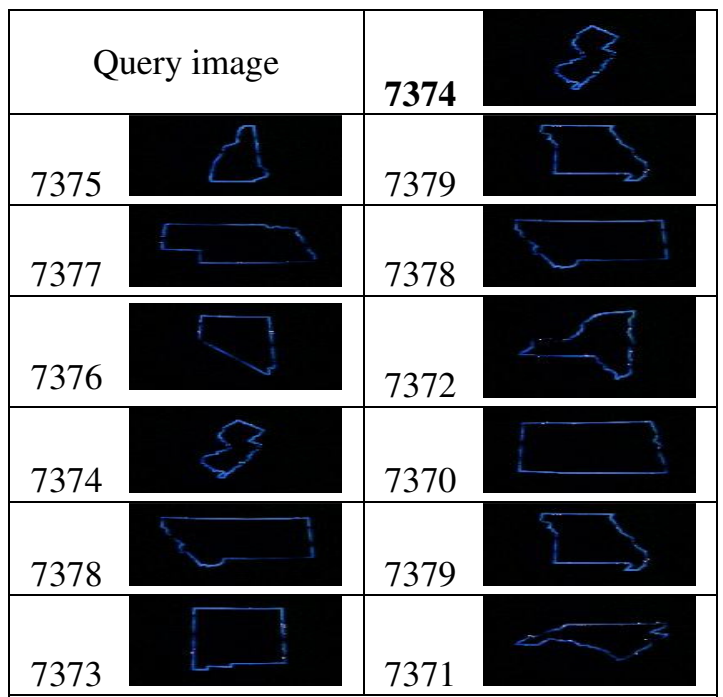

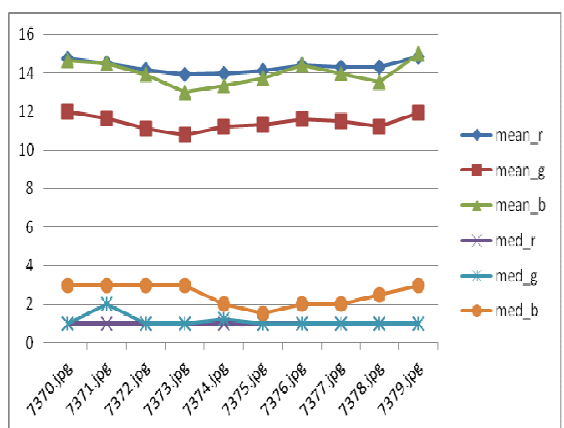

Figure 8 mean median on image set

In above graph(Figure 8) all pictures mean and median values are projected.

5.3 Selection of Huge database using median method: median red projection method is applied for the large image set, following pictures order is displayed .

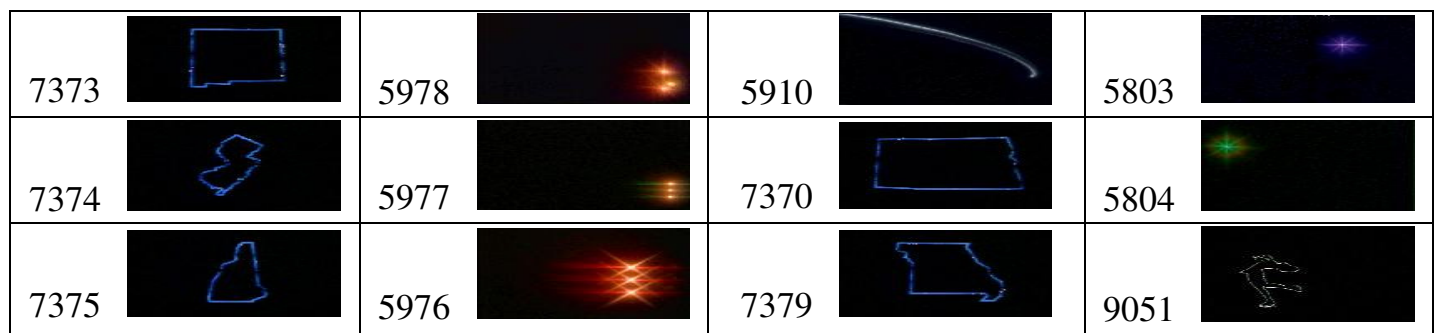


Advanced Computing: An International Journal ( ACIJ ), Vol.3, No.2, March 2012

\begin{tabular}{|l|l|l|l|l|l|l|}
\hline 7372 & & & & & & \\
\hline & & 5926 & & 5926 & & \\
\hline 5938 & & 5805 & & & \\
\hline \multicolumn{3}{|c|}{ Figure 9:Median red applied on Large set data } \\
\hline
\end{tabular}

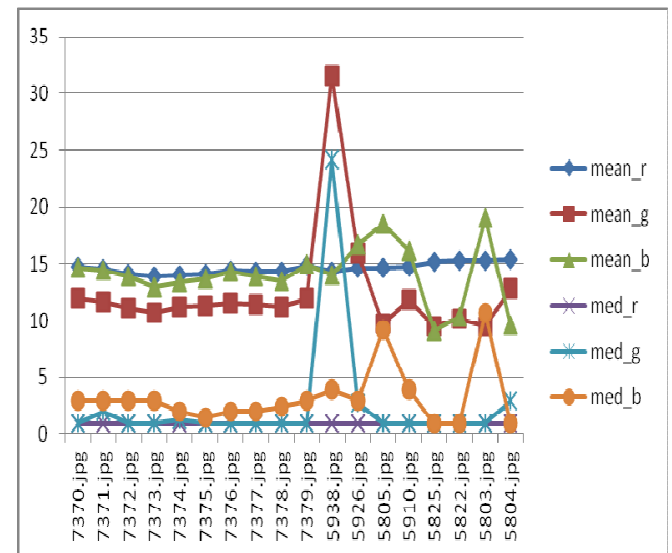

Figure 10: mean and median on image set

Large database is considered for retrieval of images .through graph(Figure 10) mean and medians method variations we can observe .at the same time pictures similarity[7][11] also changing so using proposal methods useful to find pictures similarities with efficient way.

\subsection{Standard deviation green is applied in selected set of images in which following order is performed}
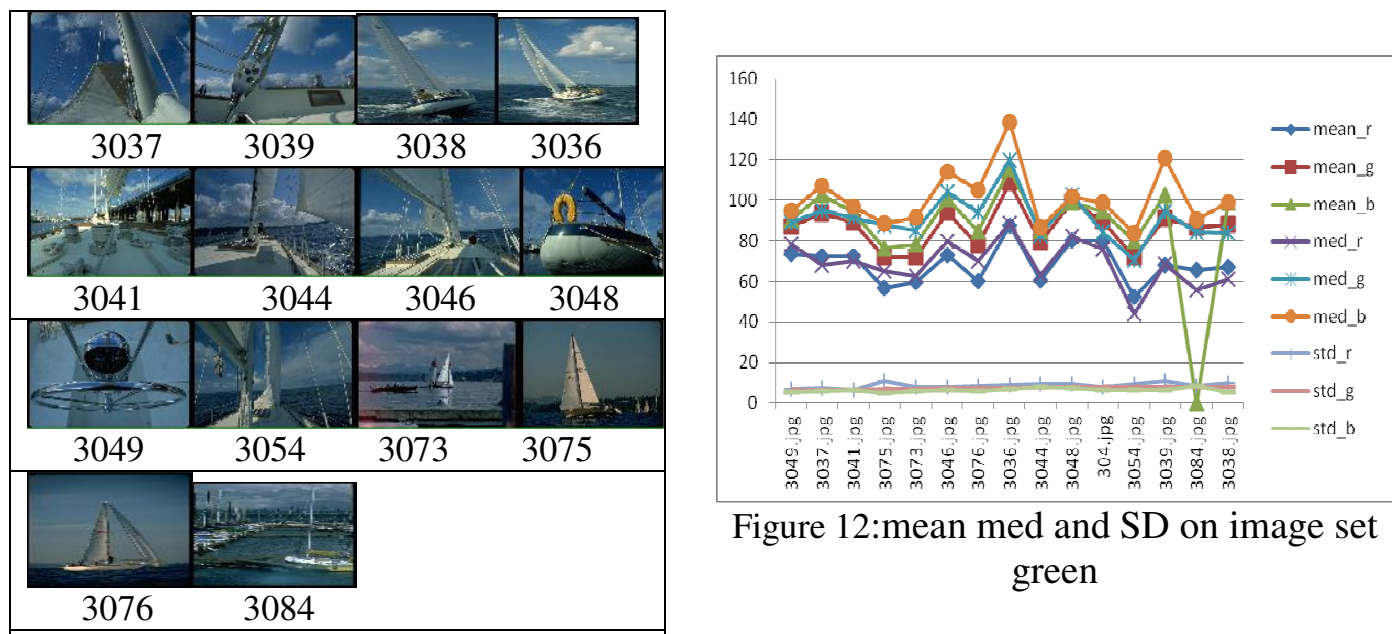

Figure 12:mean med and SD on image set

$$
\text { green }
$$

Figure 11: SD method green projection 
In Figure 11 Standard Deviation method is applied sequence of images founded in figure 12 graph showing that all method and relations between the methods on specified set of the images.

5.5 Standard deviation red is applied in selected set of images in that following order is performed
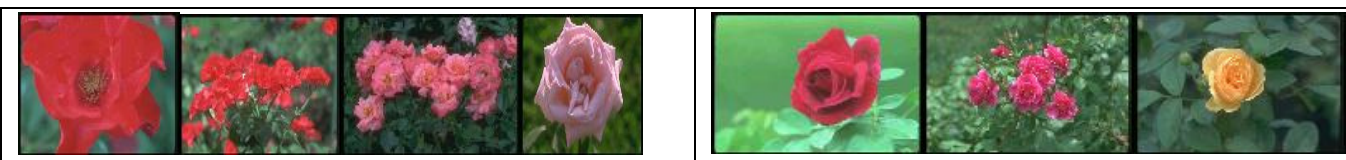

818

824

800

828

814

820

808

Figure 13: Standard deviation red is applied

\begin{tabular}{|c|c|c|c|c|c|c|c|c|c|}
\hline \multicolumn{10}{|c|}{ Table 2: Data values for comparing SD red values } \\
\hline pic name & $\begin{array}{c}\text { Mea } \\
\text { nr }\end{array}$ & $\begin{array}{c}\text { Mea } \\
\text { ng }\end{array}$ & $\begin{array}{c}\text { Me } \\
\text { anb }\end{array}$ & $\begin{array}{c}\text { Me } \\
\text { d_r }\end{array}$ & $\begin{array}{c}\text { Me } \\
\text { d_g }\end{array}$ & $\begin{array}{c}\text { Me } \\
\text { d_b }\end{array}$ & $\begin{array}{c}\text { St } \\
\text { d_r }\end{array}$ & $\begin{array}{c}\text { St } \\
\text { d_g }\end{array}$ & $\begin{array}{c}\text { St } \\
\text { d_b }\end{array}$ \\
\hline 818.jpg & 124 & 68 & 58 & 138 & 56 & 58 & 10.31 & 8.92 & 7.43 \\
\hline 824.jpg & 102 & 78 & 62 & 86 & 78 & 65 & 11.00 & 6.39 & 6.72 \\
\hline 800.jpg & 96 & 70 & 64 & 81 & 69 & 54 & 11.20 & 5.74 & 7.13 \\
\hline 828.jpg & 99 & 93 & 78 & 83 & 91 & 75 & 11.42 & 6.10 & 7.17 \\
\hline 814.jpg & 114 & 125 & 91 & 109 & 155 & 95 & 13.44 & 10.58 & 7.11 \\
\hline 820.jpg & 88 & 93 & 68 & 80 & 97 & 69 & 14.47 & 6.43 & 6.80 \\
\hline 808.jpg & 69 & 79 & 61 & 63 & 80 & 64 & 22.85 & 9.36 & 6.38 \\
\hline
\end{tabular}

Figure 14:mean ,median and SD blue method on set of images

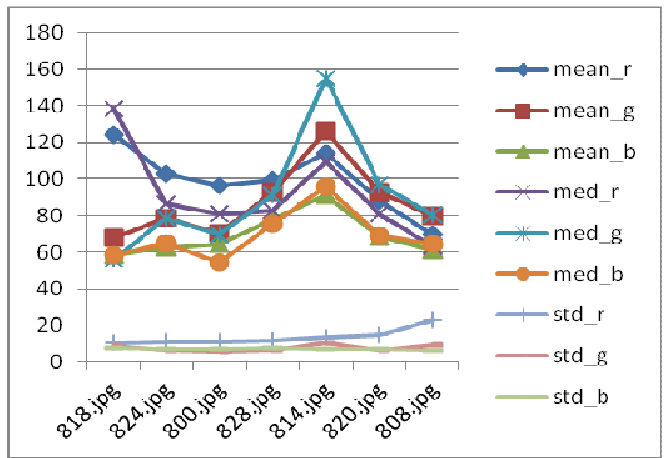

\begin{tabular}{|c|c|c|c|c|c|c|c|c|c|}
\hline \multicolumn{10}{|c|}{ Table 3: Data values for SD red ranking values } \\
\hline Fnapi cname & $\begin{array}{c}\text { Mea } \\
\text { nr }\end{array}$ & $\begin{array}{c}\text { Mea } \\
\text { ng }\end{array}$ & $\begin{array}{c}\text { Me } \\
\text { anb }\end{array}$ & $\begin{array}{c}\text { Me } \\
\text { d_r }\end{array}$ & $\begin{array}{c}\text { Me } \\
\text { d_g }\end{array}$ & $\begin{array}{c}\text { Me } \\
\text { d_b }\end{array}$ & $\begin{array}{c}\text { St } \\
\text { d_r }\end{array}$ & $\begin{array}{c}\text { St } \\
\text { d_g }\end{array}$ & $\begin{array}{c}\text { St } \\
\text { d_b }\end{array}$ \\
\hline 818.jpg & 7 & 1 & 1 & 7 & 1 & 2 & 1 & 5 & 7 \\
\hline 824.jpg & 5 & 3 & 3 & 5 & 3 & 4 & 2 & 3 & 2 \\
\hline 800.jpg & 3 & 2 & 4 & 3 & 2 & 1 & 3 & 1 & 6 \\
\hline
\end{tabular}




\begin{tabular}{|l|l|l|l|l|l|l|l|l|l|}
\hline 828.jpg & 4 & 5 & 6 & 4 & 5 & 6 & 4 & 2 & 5 \\
\hline 814.jpg & 6 & 7 & 7 & 6 & 7 & 7 & 5 & 7 & 4 \\
\hline 820.jpg & 2 & 6 & 5 & 2 & 6 & 5 & 6 & 4 & 3 \\
\hline 808.jpg & 1 & 4 & 2 & 1 & 4 & 3 & 7 & 6 & 1 \\
\hline
\end{tabular}

Above two tables are indicating feature values of $\mathrm{R} \mathrm{G} \mathrm{B} \mathrm{colors} \mathrm{of} \mathrm{images} \mathrm{.in} \mathrm{the} \mathrm{second} \mathrm{table} \mathrm{if}$ 818.jpg image is searching according to red SD, ranks are specified from 1 to 7. Similarly if any other image is searching in database with different feature selection, images display in above rank order.

5.6 Standard deviation blue is applied in selected set of images in which following order is performed and all method relations are specified through a graph.
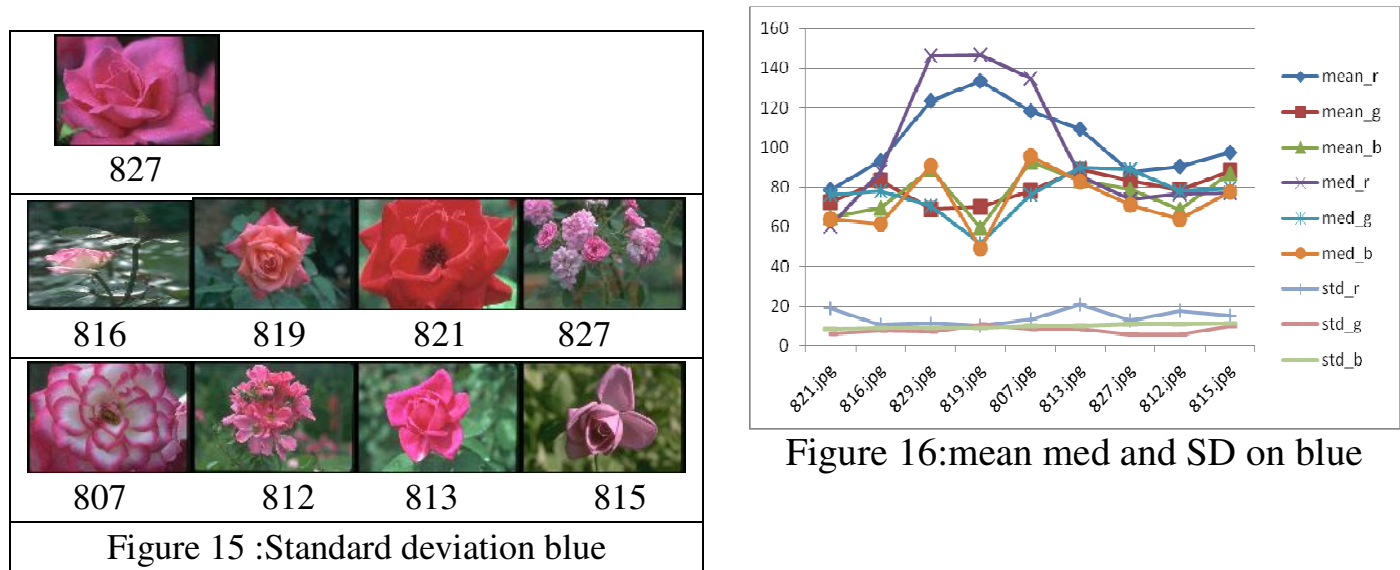

Figure 16:mean med and SD on blue

\section{FUTURE ENHANCEMENT}

Most Internet-based Content-Based Image Retrieval (CBIR) systems focus on different stock photo collections and do not address challenges of large specialized image collections and topics such as semantic[6] information retrieval by image content. in our research work we concentrated more than 10000 images with different categories of images .this work is helping towards large set of image retrieval applications. In proposed methods depending on the data set we can choose suitable methods and implement a good semantic[2] approaches in large set of images.

\section{CONCLUSION}

This paper proposes evaluation of image retrieval methods All proposed method are shown different comparison of the obtained results with other approach demonstrates that the proposed approach improves accuracy rate comparatively existed methods which is used to retrieve the images from the large database .in this work we compared total 10000 images with different categories. All suggested methods are helpful to perform the good results and based on query images what are the images retrieved from the database ,all images showing vary similar and from method to method results are showing variation so best one is to select the combinations of colors mean with median and standard deviation, So we can expect using proposed methods best performs and good results. Current techniques are based on low level features and there is a huge semantic gap. In future days more research work is required in semantically competent systems. 


\section{REFERENCES}

[1] R.Datta, D.Joshi, J.Li, J.Z.Wang. "Image Retrieval: ideas, Influences, and Trends of the New Age". ACM Transactions on Computing Surveys, Vol. 40, No. 2(2008)

[2] Najlae Idrissi. "Bridging the Semantic Gap for Texture based Image Retrieval and Navigation, Journal Of Multimedia”, Vol.4, No.5, October 2009

[3] M. L. Kherfi, D. Ziou, and A. Benard. "Image retrieval from the World Wide Web: Issues, techniques and systems". ACM Computing Surveys, 36(1):35\{67, 2004\}

[4] A. W. M. Smeulders, M. Worring, S. Santini, A. Gupta, and R. Jain. "Content based image retrieval at the end of the early years". IEEE Transactions on Pattern Analysis and Machine Intelligence, 22(12):1349 1380, December 2000.

[5]Alham, N.K.; Maozhen Li; Hammoud, S.; Hao Qi; , "Evaluating Machine Learning Techniques for Automatic Image Annotations," Fuzzy Systems and Knowledge Discovery, 2009. FSKD '09. Sixth International Conference on , vol.7, no., pp.245-249, 14-16 Aug. 2009

[6] Smeulders, A.W.M., Worring, M., Santini, S., Gupta, A., Jain, R. "Content based image retrieval at the end of the early years". IEEE Trans. Pattern Anal. Mach. Intell. 22 1349-1380 (2000)

[7] Wei Jiang, Kap luk chan, Mingjing Li. „, "apping low level features to high level semantic concept in region based image retrieval ", IEEE CVPR05. 2005

[7] Vieux, R.; Benois-Pineau, J.; Domenger, J.-P.; Braquelaire, A.; , "ESPI Image Indexing and Similarity Search in Radon Transform Domain," Content-Based Multimedia Indexing, 2009. CBMI '09. Seventh International Workshop on , vol., no., pp.231-236, 3-5 June 2009doi: 10.1109/CBMI.2009.38

[9] Huan Wang, Song Liu, Liang Tien Chia. "Image retrieval with a multi modality ontology". Multimedia System (2008)

[10] Deb, S.; , "Overview of research on finding semantic meanings from low-level features in contentbased image retrieval," Pervasive Computing (JCPC), 2009 Joint Conferences on, vol., no., pp.203-208, 3-5 Dec. 2009.

[11] Mofaddel, M.A.; Sadek, S.; , "Adult image content filtering: A statistical method based on MultiColor Skin Modeling," Computer Technology and Development (ICCTD), 2010 2nd International Conference on , vol., no., pp.744-748, 2-4 Nov. 2010 doi: 10.1109/ICCTD.2010.5646416

[12] Tungkasthan, A.; Intarasema, S.; Premchaiswadi, W.; , "Spatial color indexing using ACC algorithm," ICT and Knowledge Engineering, 2009 7th International Conference on , vol., no., pp.113117, 1-2 Dec. 2009 doi: 10.1109/ICTKE.2009.5397321

[13] R.Venkata Ramana Chary, Dr. D. Rajya Lakshmi, Dr. K.V.N. Sunitha Published an International Journal on "Image Retrieval and Similarity Measurement Based on Image Feature" in International Journal of Computer Science and Technology (IJCST) Volume 2 ,Issue 4 OCT-DEC 2011.PP385-389 ISSN :0976-8491(ONLINE)IISSN:2229-4333(PRINT).

[14] R.Venkata Ramana Chary, Dr. K.V.N. Sunitha, Dr. D. Rajya Lakshmi Published an International journal "Quality Measures on Multispectral Images" in international journal of Image Processing and Applications(IJIPA) ,volume 2(1),2011,July- December 2011 .pp 101-105 ISSN:0975-8178

[15] Ion, A.; Stanescu, L.; Burdescu, D.; Udristoiu, S.; , "Mapping Image Low-Level Descriptors to Semantic Concepts," Computing in the Global Information Technology, 2008. ICCGI '08. The Third International Multi-Conference on , vol., no., pp.154-159, July 27 2008-Aug. 12008.

[16]Tseng, V.S.; Ming-Hsiang Wang; Ja-Hwung Su; , "A New Method for Image Classification by Using Multilevel Association Rules," Data Engineering Workshops, 2005. 21st International Conference on , vol., no., pp. 1180, 05-08 April 2005 


\section{Authors}

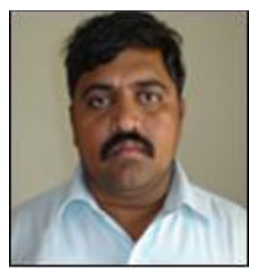

R.Vekata Ramana Chary is working as a Associate Professor in the Department of CSE at Padmasri Dr. B.V.Raju Institute of Technology, Narsapur, Medak, AP, India. Presently he is pursuing Ph.D from GITAM University, AP, India. His research interest is in Image Processing, Performance Measurements, Algorithms Analysis and Programming Techniques. He is having 15 years of teaching and research experience

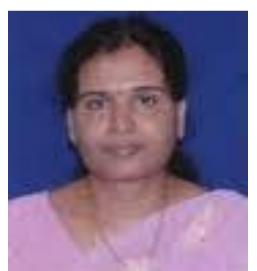

Dr. D. Rajya Lakshmi is working as Professor and HOD in the Department of IT at GITAM University, Visakhapatnam, AP, India. Her research areas include Image processing and Data mining. She is having about 17 years of teaching and research experience. Her $\mathrm{PhD}$ was awarded from JNTUH, Hyderabad, AP in the area of Image Processing

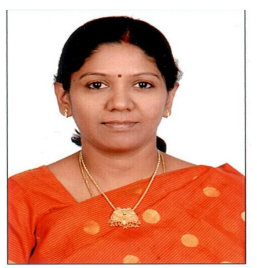

Dr. K.V.N. Sunitha is working as a Professor and HOD in Department of CSE at G. Narayanamma Institute Of Technology and Science (for WOMEN) in Hyderabad, AP, India. Her PhD was awarded from JNTUH Hyderabad AP. She is having 18 years of teaching and research experience in the Computer Science field. Her research interest is in Compilers, NLP, Speech Recognition, Computer Networks and Image Processing. 